\title{
ENHANCED PHOTONIC BAND GAPS OF PERIODIC DIELECTRIC STRUCTURES COMPOSED OF DOUBLE CYLINDRICAL RODS
}

\author{
T. Woźniak, M. Krawczyk and H. Puszkarski* \\ Surface Physics Division, Faculty of Physics, Adam Mickiewicz University \\ Umultowska 85, 61-614 Poznań, Poland
}

(Received February 19, 2001)

\begin{abstract}
On the basis of Maradudin and McGurn's model we investigate the spectral properties of a two-dimensional photonic crystal, consisting of cylindrical rods disposed periodically throughout a dielectric matrix (the crystal is limited by two surfaces perpendicular to the axes of the rods). The model is supplemented by a new structural element in the form of dielectric rods (also cylindrical in shape) disposed coaxially within the original rods. The centers of both kinds of rods overlap forming a two-dimensional rectangular lattice in the plane parallel to the surface. Thus, the object of our study is a Maradudin-McGurn's model in which the individual rods are replaced by coaxial double rods. For this modified model we search for the optimal set of structural parameter values for which the forbidden energy gaps become greatest, giving especial attention to the role played by the additional inner rods in the emergence of gaps. We arrive at the conclusion that the condition for the opening of absolutely forbidden gaps become more favourable in a photonic crystal with double rods compared with those required in a crystal with simple rods.

PACS numbers: 78.66.Sq, 78.66.-w, 42.25.Bs
\end{abstract}

\section{Introduction}

Following the publication of Yablonovitch [1], reporting experimentally the existence of energy gaps in spectrum of the electromagnetic waves propagating in a periodic dielectric structure (referred to as photonic crystal), there appeared several papers reporting calculations of the photonic band structures in periodic systems [2-7] differing by the types of inclusions embedded periodically into the background matrix. Especial interest has been devoted to the study of two-dimensional (2D) systems, consisting of parallel homogeneous rods [8-25], having different

* corresponding author; e-mail: henpusz@main.amu.edu.pl 
shapes of their cross-sections and embedded in a homogeneous matrix with properties differing from those of the rods. Recently, the paper has appeared [26], in which the new structural element has been included into the $2 \mathrm{D}$ photonic crystal model, namely the authors considered nonhomogeneous (dielectric-metallic) rods instead of the purely dielectric ones. Following this line, in this work we also consider the $2 \mathrm{D}$ periodic composite, in which the single dielectric rods are replaced by coaxially-double dielectric rods (cylindrical in shape) and we search for the optimal set of structural parameter values for which the forbidden energy gaps become greatest: we give especial attention to the role played by the additional inner rods in the emergence of gaps.

After the presentation of the general theory of 2D photonic crystals in Sec. 2 , we proceed to the theoretical analysis of the structure consisting of single rods (Sec. 3), and afterwards - consisting of double rods (Sec. 4). The calculated respective photonic energy spectra are presented in Sec. 5, and finally Sec. 6 presents the conclusions drawn from our calculations.

\section{The model of a two-dimensional photonic crystal}

As standard, we choose the two-dimensional model proposed by Maradudin and McGurn [27], where the photonic crystal consists of a periodic lattice composed of identical parallel rods (characterized by a dielectric constant $\varepsilon_{a}$ ) disposed in a homogeneous dielectric matrix (with dielectric constant $\varepsilon_{b}$ ). The dielectric rods are parallel to the $x_{3}$-axis, whereas the crystal as a whole is limited by two metallic planes perpendicular to the $x_{3}$-axis, disposed in the points $x_{3}=0$ and $x_{3}=d$ (see Fig. 1). In the first place, we shall study the case of a structure with cylindrical rods (the case already thoroughly analyzed in the literature [19-22]). Obviously, the cross-sections of the rods will form on the plane perpendicular to the $x_{3}$-axis a square two-dimensional lattice and positions of the nodes of the lattice are given by the two-dimensional vector

$$
x_{\|}(l)=l_{1} a_{1}+l_{2} a_{2},
$$

where coefficients $l_{1}$ and $l_{2}$ assume arbitrary integer values; the symbol $\|$ denotes a two-dimensional vector parallel to the $x_{1}, x_{2}$-plane. The dielectric constant $\varepsilon(x)$ of the composite can be expressed as dependent on the position vector $x_{\|}$:

$$
x_{\|}=\widehat{x}_{1} x_{1}+\widehat{x}_{2} x_{2}
$$

(where $\widehat{x}_{1}$ and $\widehat{x}_{2}$ are direction versors of the axes $x_{1}$ and $x_{2}$, respectively) being a periodic function of $x_{\|}$. This can be described by the relation

$$
\varepsilon\left[x_{\|}+x_{\|}(l)\right]=\varepsilon\left(x_{\|}\right),
$$

where $x_{\|}(l)$ is an arbitrary two-dimensional vector of the crystal lattice defined by Eq. (1).

We introduce the reciprocal lattice defined by

$$
\boldsymbol{G}_{\|}(h)=h_{1} \boldsymbol{b}_{1}+h_{2} \boldsymbol{b}_{2} \text {, }
$$

where $h_{1}$ and $h_{2}$ are arbitrary integers, and the basis vectors of the two-dimensional reciprocal lattice $\boldsymbol{b}_{1}$ and $\boldsymbol{b}_{2}$ are defined by the standard relations

$$
\boldsymbol{a}_{i} \cdot \boldsymbol{b}_{j}=2 \pi \delta_{i j}
$$




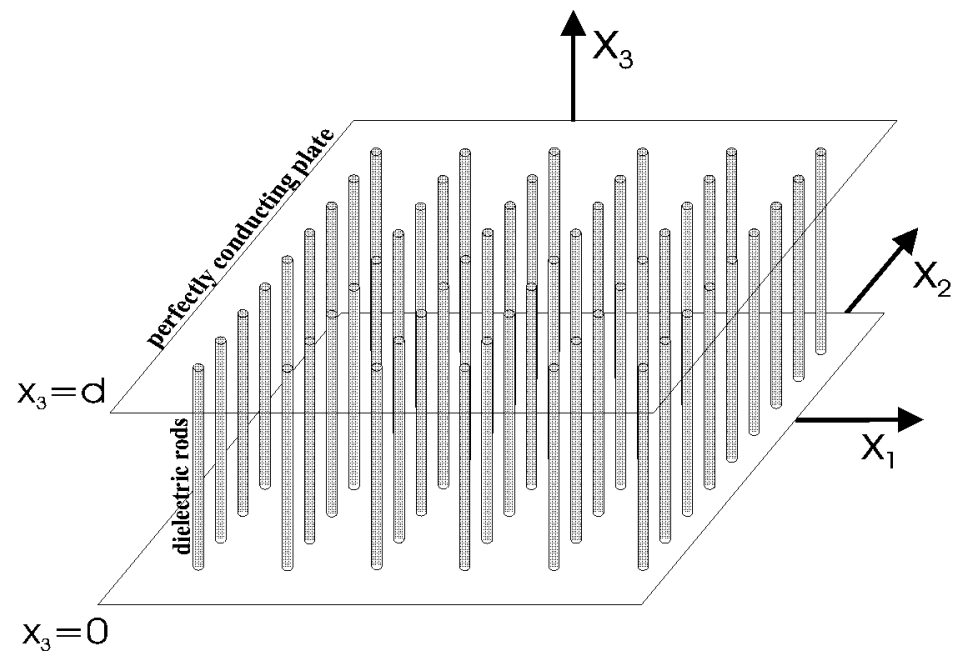

Fig. 1. Model of a two-dimensional photonic crystal limited by two perfectly conducting surfaces perpendicular to axes of the rods (after Maradudin and McGurn [27]).

On introducing the basic definitions related to the structure under consideration we make use of the equation which has to be fulfilled by the electromagnetic field $\boldsymbol{E}$

$$
\nabla \times \nabla \times \boldsymbol{E}(\boldsymbol{x} \mid \omega)=\varepsilon\left(\boldsymbol{x}_{\|}\right) \frac{\omega^{2}}{c^{2}} \boldsymbol{E}(\boldsymbol{x} \mid \omega) .
$$

Equation (6) gives the relations between the electric field components. Therefore, the following three equations:

$$
\begin{aligned}
& \frac{1}{\varepsilon\left(x_{\|}\right)}\left(-\frac{\partial^{2} E_{1}}{\partial x_{2}^{2}}-\frac{\partial^{2} E_{1}}{\partial x_{3}^{2}}+\frac{\partial^{2} E_{2}}{\partial x_{1} \partial x_{2}}+\frac{\partial^{2} E_{3}}{\partial x_{1} \partial x_{3}}\right)=\frac{\omega^{2}}{c^{2}} E_{1}, \\
& \frac{1}{\varepsilon\left(x_{\|}\right)}\left(\frac{\partial^{2} E_{1}}{\partial x_{2} \partial x_{1}}-\frac{\partial^{2} E_{2}}{\partial x_{3}^{2}}-\frac{\partial^{2} E_{2}}{\partial x_{1}^{2}}+\frac{\partial^{2} E_{3}}{\partial x_{2} \partial x_{3}}\right)=\frac{\omega^{2}}{c^{2}} E_{2} \\
& \frac{1}{\varepsilon\left(x_{\|}\right)}\left(\frac{\partial^{2} E_{1}}{\partial x_{3} \partial x_{1}}+\frac{\partial^{2} E_{2}}{\partial x_{3} \partial x_{2}}-\frac{\partial^{2} E_{3}}{\partial x_{1}^{2}}-\frac{\partial^{2} E_{3}}{\partial x_{2}^{2}}\right)=\frac{\omega^{2}}{c^{2}} E_{3}
\end{aligned}
$$

describe the propagation of a wave in an inhomogeneous medium with position-dependent dielectric constant $\varepsilon\left(\boldsymbol{x}_{\|}\right)$. In the case of the photonic crystal model introduced above, we still have to consider the role of the metallic planes bounding the crystal. They impose boundary conditions annihilating the tangential components of the electromagnetic field on the surfaces. We write those conditions as follows:

$$
E_{1}=E_{2}=0 \text { for } x_{3}=0 ; d .
$$

Combining (7), (8) and (9) and the boundary conditions (10) and using Bloch's theorem we obtain

$$
E_{1}(x \mid \omega)=\sum_{\mathbb{G}_{\|}^{n}} \sum_{n=1}^{\infty} a_{1}^{(n)}\left(\boldsymbol{k}_{\|}+\boldsymbol{G}_{\|}\right) \mathrm{e}^{\left[\mathrm{i}\left(\boldsymbol{k}_{\|}+\mathbb{G}_{\|}\right) \cdot \boldsymbol{x}_{\|]}\right]} \sin \left(\frac{n \pi x_{3}}{d}\right),
$$




$$
\begin{aligned}
& E_{2}(x \mid \omega)=\sum_{\mathbb{G}_{\|}} \sum_{n=1}^{\infty} a_{2}^{(n)}\left(\boldsymbol{k}_{\|}+\boldsymbol{G}_{\|}\right) \mathrm{e}^{\left[\mathrm{i}\left(\boldsymbol{k}_{\|}+\mathbb{G}_{\|}\right) \cdot \boldsymbol{x}_{\|]}\right]} \sin \left(\frac{n \pi x_{3}}{d}\right), \\
& E_{3}(x \mid \omega)=\mathrm{i} \sum_{\mathbb{G}_{\|}} \sum_{n=0}^{\infty} \varepsilon_{n} a_{3}^{(n)}\left(\boldsymbol{k}_{\|}+\boldsymbol{G}_{\|}\right) \mathrm{e}^{\left[\mathrm{i}\left(\boldsymbol{k}_{\|}+\mathbb{G}_{\|} \|\right) \cdot \boldsymbol{x}_{\|]}\right]} \cos \left(\frac{n \pi x_{3}}{d}\right),
\end{aligned}
$$

where $\boldsymbol{k}_{\|}=\widehat{x}_{1} k_{1}+\widehat{x}_{2} k_{2}$ is a two-dimensional wave vector, and $\varepsilon_{n}$ (resulting from the definition of the expansion in a Fourier series) is given as

$$
\varepsilon_{n}=\left\{\begin{array}{cll}
1 / 2 & \text { for } & n=0 \\
1 & \text { for } & n \geq 1
\end{array}\right.
$$

The inverse dielectric permittivity is a periodic function also with a period given by that of the Bravais lattice, thus

$$
\frac{1}{\varepsilon\left(x_{\|}\right)}=\frac{1}{\varepsilon\left(x_{\|}+x_{\|}(l)\right)} \text {. }
$$

Accordingly, we are justified in carrying out the expansion

$$
\frac{1}{\varepsilon\left(x_{\|}\right)}=\sum_{G_{\|}^{*}} \widehat{\kappa}\left(\boldsymbol{G}_{\|}\right) \mathrm{e}^{\mathrm{i} \boldsymbol{G}_{\|} \| x_{\|}},
$$

where the summation extends over all the vectors $\boldsymbol{G}_{\|}$of the reciprocal lattice. The respective coefficients of the Fourier expansion are given by the formula

$$
\widehat{\kappa}\left(\boldsymbol{G}_{\|}\right)=\frac{1}{V_{c}} \int_{c} \mathrm{~d} \boldsymbol{x}_{\|} \mathrm{e}^{-\mathrm{i} \mathbb{G}_{\|\|} \cdot x_{\|}} \frac{1}{\varepsilon\left(\boldsymbol{x}_{\|}\right)},
$$

where integration extends over an arbitrary primitive elementary cell $c$ of the simple lattice and $V_{c}$ is its volume. On inserting (11) and (13) into (7), (8) and (9) we obtain equations for the coefficients $a_{\alpha}^{(n)}\left(k_{\|}+\boldsymbol{G}_{\|}\right), \alpha=1,2,3 ; n=0,1,2, \ldots$

Let us start from the case $n=0$ (the first harmonic). For this case the set of Eqs. (7), (8) and (9) is reduced to a single equation since for $n=0$ the components $E_{1}(x \mid \omega)$ and $E_{2}(x \mid \omega)$ vanish (see Eq. (11) - the function $\sin \left(n \pi x_{3} / d\right)$ vanishes for $n=0)$ :

$$
\frac{1}{\varepsilon\left(x_{\|}\right)}\left(-\frac{\partial^{2} E_{3}}{\partial x_{1}^{2}}-\frac{\partial^{2} E_{3}}{\partial x_{2}^{2}}\right)=\frac{\omega^{2}}{c^{2}} E_{3}
$$

On inserting the simplified equation for the $E_{3}$-component of the electric field and the Fourier transform of the function $\varepsilon^{-1}\left(x_{\|}\right)$into (15), together with (11) and (13) we obtain

$$
\sum_{\boldsymbol{G}_{\|\|}^{\prime \prime}} \widehat{\kappa}\left(\boldsymbol{G}_{\|}-\boldsymbol{G}_{\|}^{\prime \prime}\right) a_{3}^{(0)}\left(\boldsymbol{k}_{\|}+\boldsymbol{G}_{\|}^{\prime \prime}\right)\left(\boldsymbol{k}_{\|}+\boldsymbol{G}_{\|}^{\prime \prime}\right)^{2}=\frac{\omega^{2}}{c^{2}} a_{3}^{(0)}\left(\boldsymbol{k}_{\|}+\boldsymbol{G}_{\|}\right)
$$

— an equation for the coefficients $a_{3}^{(0)}\left(\boldsymbol{k}_{\|}+\boldsymbol{G}_{\|}\right)$. This is a standard form of the equation for the eigenvalues of the symmetric matrix which holds for an arbitrary vector $\boldsymbol{G}_{\|}$of the reciprocal lattice.

Our further calculations require the knowledge of the explicit form of the coefficient $\widehat{\kappa}\left(\boldsymbol{G}_{\|}\right)$, the Fourier component of the function $\varepsilon\left(\boldsymbol{x}_{\|}\right)^{-1}$ (see (13) and (14)). 
It is noteworthy that we are always justified in writing the following definition of the dielectric permittivity $\varepsilon\left(\boldsymbol{x}_{\|}\right)^{-1}$ irrespective of the shape of the rod cross-section (see Ref. [16])

$$
\frac{1}{\varepsilon\left(x_{\|}\right)}=\frac{1}{\varepsilon_{b}}+\left(\frac{1}{\varepsilon_{a}}-\frac{1}{\varepsilon_{b}}\right) \sum_{l_{1}=-\infty}^{\infty} \sum_{l_{2}=-\infty}^{\infty} S\left[\boldsymbol{x}_{\|}-x_{\|}(l)\right],
$$

where:

$$
S\left(\boldsymbol{x}_{\|}\right)= \begin{cases}1 & \text { if vector } \boldsymbol{x}_{\|} \text {denotes a point situated inside the rod, } \\ 0 & \text { if it is situated outside the rod. }\end{cases}
$$

On making use of the above definition and Eq. (14) we obtain the following formula for the expansion coefficient $\hat{\kappa}\left(\boldsymbol{G}_{\|}\right)$for the two-dimensional square lattice $\left(a_{1}=\right.$ $\left.a_{2}=a\right)$ :

$$
\begin{gathered}
\widehat{\kappa}\left(\boldsymbol{G}_{\|}\right)=\frac{1}{a^{2}} \int_{-\frac{1}{2} a}^{\frac{1}{2} a} \mathrm{~d} x_{1} \int_{-\frac{1}{2} a}^{\frac{1}{2} a} \mathrm{~d} x_{2}\left[\frac{1}{\varepsilon_{b}}-\left(\frac{1}{\varepsilon_{a}}-\frac{1}{\varepsilon_{b}}\right) \sum_{l_{1}, l_{2}=-\infty}^{\infty} S\left[x_{\|}-x_{\|}(l)\right]\right] \\
\times \mathrm{e}^{-\mathrm{i} \mathbb{G}_{\|} \| x_{\|}}=\underbrace{\frac{1}{\varepsilon_{b}} \frac{1}{a^{2}} \int_{-\frac{1}{2} a}^{\frac{1}{2} a} \mathrm{~d} x_{1} \int_{-\frac{1}{2} a}^{\frac{1}{2} a} \mathrm{~d} x_{2} \mathrm{e}^{-\mathrm{i} \mathbb{\pi}_{\|} \| x_{\|}}}_{A} \\
-\underbrace{\frac{1}{a^{2}} \int_{-\frac{1}{2} a}^{\frac{1}{2} a} \mathrm{~d} x_{1} \int_{-\frac{1}{2} a}^{\frac{1}{2} a} \mathrm{~d} x_{2}\left(\frac{1}{\varepsilon_{a}}-\frac{1}{\varepsilon_{b}}\right) \sum_{l_{1}, l_{2}=-\infty}^{\infty} S\left[x_{\|}-x_{\|}(l)\right] \mathrm{e}^{-\mathrm{i} \mathbb{G}_{\|} \| \cdot x_{\|}}}_{B}
\end{gathered}
$$

We now proceed to consider the integrals denoted above as $A$ and $B$. Regarding the first one, we invoke the properties of the Dirac function $\delta$

$$
\delta(x)=0 \quad \text { for } \quad x \neq 0 \quad \text { and } \quad \int \delta(x) \mathrm{d} x=1
$$

(the range of integration comprises the point $x=0$ ). A particularly useful representation of the function $\delta$ is based on the function $(\sin g x) / \pi x$, where $g$ is an arbitrary real number

$$
\delta(x)=\lim _{g \rightarrow \infty} \frac{\sin g x}{\pi x} .
$$

We consider the integral

$$
\begin{gathered}
\frac{1}{2 \pi} \int_{-\infty}^{\infty} \mathrm{e}^{-\mathrm{i} k x} \mathrm{~d} x=\lim _{g \rightarrow \infty} \frac{1}{2 \pi} \int_{-g}^{g} \mathrm{e}^{-\mathrm{i} k x} \mathrm{~d} x=\lim _{g \rightarrow \infty} \frac{1}{2 \pi} \int_{-g}^{g}[\cos (k x)+\mathrm{i} \sin (k x)] \mathrm{d} x \\
=\lim _{g \rightarrow \infty} \frac{1}{2 \pi} \int_{-g}^{g} \cos (k x) \mathrm{d} x+\lim _{g \rightarrow \infty} \frac{1}{2 \pi} \int_{-g}^{g} \mathrm{i} \sin (k x) \mathrm{d} x \\
=\left.\lim _{g \rightarrow \infty} \frac{1}{2 \pi} \frac{1}{k} \sin (k x)\right|_{-g} ^{g}-\underbrace{\left.\lim _{g \rightarrow \infty} \frac{1}{2 \pi} \frac{1}{k} \mathrm{i} \cos (k x)\right|_{-g} ^{g}}_{=0}
\end{gathered}
$$




$$
=\lim _{g \rightarrow \infty} \frac{1}{2 \pi} \frac{1}{k}[\sin (k g)-\sin (-g k)]=\lim _{g \rightarrow \infty} \frac{2 \sin (k g)}{2 \pi k}=\lim _{g \rightarrow \infty} \frac{\sin (k g)}{\pi k}=\delta(k) ;
$$

finally

$$
\frac{1}{2 \pi} \int_{-\infty}^{\infty} \mathrm{e}^{-\mathrm{i} k x} \mathrm{~d} x=\delta(k) .
$$

Thus, the integral $A$ (see formula (19)) amounts to

$$
A=\frac{1}{\varepsilon_{b}} \frac{1}{a^{2}} \int_{c} \mathrm{e}^{-\mathrm{i} \boldsymbol{G}_{\|} \cdot x_{\|}}=\frac{1}{\varepsilon_{b}} \delta\left(\boldsymbol{G}_{\|}\right) .
$$

Regarding the integral $B$ (see formula (20)) we perform integration over the range of the elementary cell whereas summation ranges over all the nodes of the real lattice defined by the vector $x_{\|}(l)$ :

$$
\begin{aligned}
& B=\frac{1}{a^{2}} \int_{-\frac{1}{2} a}^{\frac{1}{2} a} \mathrm{~d} x_{1} \int_{-\frac{1}{2} a}^{\frac{1}{2} a} \mathrm{~d} x_{2}\left(\frac{1}{\varepsilon_{a}}-\frac{1}{\varepsilon_{b}}\right) \sum_{l_{1}, l_{2}=-\infty}^{\infty} S\left[x_{\|}-x_{\|}(l)\right] \mathrm{e}^{-i \mathbb{G}_{\|} \cdot \boldsymbol{x}_{\|}} \\
& =\frac{1}{a^{2}}\left(\frac{1}{\varepsilon_{a}}-\frac{1}{\varepsilon_{b}}\right) \sum_{l_{1}, l_{2}=-\infty}^{\infty} \int_{-\frac{1}{2} a}^{\frac{1}{2} a} \mathrm{~d} x_{1} \int_{-\frac{1}{2} a}^{\frac{1}{2} a} \mathrm{~d} x_{2} S\left[x_{\|}-x_{\|}(l)\right] \mathrm{e}^{-\mathrm{i} \mathbb{G}_{\|} \| x_{\|}} .
\end{aligned}
$$

In Eq. (25) the sum over $l_{1}$ and $l_{2}$ together with the double integral over $\mathrm{d} x_{1}$ and $\mathrm{d} x_{2}$ is constructed so that the vector $x_{\|}-x_{\|}(l)$, which is the argument of the function $S$, shall span all the points of the plane $x_{1} x_{2}$. Following the procedure of [16], we perform a transition consisting in a change of the integration limits $-\frac{1}{2} a$ to $-\infty$ and $\frac{1}{2} a$ to $\infty$ simultaneously transferring the vector $x_{\|}(l)$ into the origin of coordinates (an operation equivalent to the liquidation of summation over $l_{1}$ and $l_{2}$ ). The above transformation gives

$$
B=\frac{1}{a^{2}}\left(\frac{1}{\varepsilon_{a}}-\frac{1}{\varepsilon_{b}}\right) \int_{-\infty}^{\infty} \mathrm{d} x_{1} \int_{-\infty}^{\infty} \mathrm{d} x_{2} S\left(x_{\|}\right) \mathrm{e}^{-\mathrm{i} G_{\|} \cdot x_{\|}}
$$

which inserted into (20) leads finally to an expression that will hold for an arbitrary shape of the cross-section of the dielectric rods

$$
\widehat{\kappa}\left(\boldsymbol{G}_{\|}\right)=\frac{1}{\varepsilon_{b}} \delta\left(\boldsymbol{G}_{\|}\right)+\frac{1}{a^{2}} \int_{-\infty}^{\infty} \mathrm{d} x_{1} \int_{-\infty}^{\infty} \mathrm{d} x_{2}\left(\frac{1}{\varepsilon_{a}}-\frac{1}{\varepsilon_{b}}\right) S\left(\boldsymbol{x}_{\|}\right) \mathrm{e}^{-\mathrm{i} \mathbb{G}_{\|} \cdot \boldsymbol{x}_{\|}} .
$$

On distinguishing between the two cases $\boldsymbol{G}_{\|}=0$ and $\boldsymbol{G}_{\|} \neq 0$, Eq. (27) can be rewritten in the final form

$$
\widehat{\kappa}\left(\boldsymbol{G}_{\|}\right)= \begin{cases}\frac{1}{\varepsilon_{b}}+\frac{1}{a^{2}} \int_{-\infty}^{\infty} \mathrm{d} x_{1} \int_{-\infty}^{\infty} \mathrm{d} x_{2}\left(\frac{1}{\varepsilon_{a}}-\frac{1}{\varepsilon_{b}}\right) S\left(x_{\|}\right) & \text {for } \boldsymbol{G}_{\|}=0, \\ \frac{1}{a^{2}} \int_{-\infty}^{\infty} \mathrm{d} x_{1} \int_{-\infty}^{\infty} \mathrm{d} x_{2}\left(\frac{1}{\varepsilon_{a}}-\frac{1}{\varepsilon_{b}}\right) S\left(\boldsymbol{x}_{\|}\right) \mathrm{e}^{-\mathrm{i} \boldsymbol{G}_{\|} \cdot x_{\|}} & \text {for } \boldsymbol{G}_{\|} \neq 0 .\end{cases}
$$




\section{The case of single cylindrical rods}

In order to make Eq. (28) applicable for the determination of the quantity $\widehat{\kappa}\left(\boldsymbol{G}_{\|}\right)$in the case of a photonic crystal with rods having a circular cross-section (see Fig. 2), we have to express the function $S\left(x_{\|}\right)$, occurring in the preceding

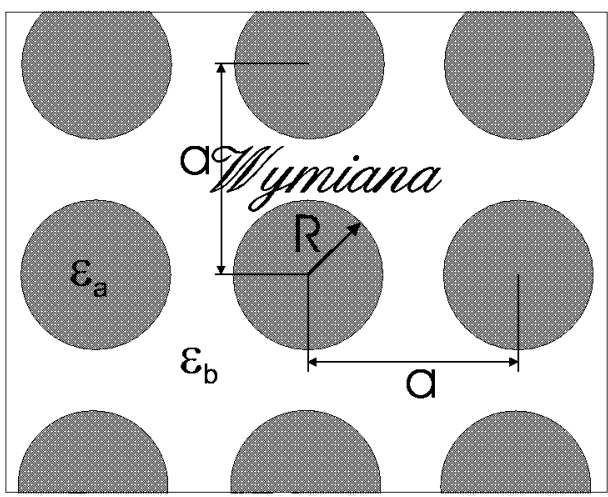

Fig. 2. The top view of the $2 \mathrm{D}$ square array of single circular rods; $\varepsilon_{a}$ and $\varepsilon_{b}$ denote, respectively, dielectric constants of the rods and the background, $a$ and $R$ represent, respectively, the lattice constant and the radius of the rods.

formulae, explicitly in terms of the parameters of the structure under consideration

$$
S\left(x_{\|}\right)=\left\{\begin{array}{lll}
1 & \text { for } & \left|x_{\|}\right| \leq R, \\
0 & \text { for } & \left|x_{\|}\right|>R,
\end{array}\right.
$$

where $R$ is the radius of the circle determined by the cross-section of the rod with a plane perpendicular to the axis. On inserting into (27) and going over to spherical coordinates, we obtain

$$
\widehat{\kappa}\left(\boldsymbol{G}_{\|}\right)=\frac{1}{\varepsilon_{b}} \delta\left(\boldsymbol{G}_{\|}\right)+\frac{1}{a^{2}}\left(\frac{1}{\varepsilon_{a}}-\frac{1}{\varepsilon_{b}}\right) \int_{0}^{\infty} \mathrm{d} r \int_{0}^{2 \pi} \mathrm{d} \varphi r S\left(\boldsymbol{x}_{\|}\right) \mathrm{e}^{-\mathrm{i} G_{\|} r \cos \varphi} .
$$

Since the function $S\left(x_{\|}\right)$vanishes everywhere except inside the rod (see (29)), we get the following expression for the coefficient $\widehat{\kappa}\left(\boldsymbol{G}_{\|}\right)$:

$$
\widehat{\kappa}\left(\boldsymbol{G}_{\|}\right)=\frac{1}{\varepsilon_{b}} \delta\left(\boldsymbol{G}_{\|}\right)+\frac{1}{a^{2}}\left(\frac{1}{\varepsilon_{a}}-\frac{1}{\varepsilon_{b}}\right) \int_{0}^{R} \mathrm{~d} r \int_{0}^{2 \pi} \mathrm{d} \varphi r \mathrm{e}^{-\mathrm{i} G_{\|} r \cos \varphi},
$$

where $G_{\|}$is the length of the vector $\boldsymbol{G}_{\|}$. In order to calculate the above integral we have recourse to the Bessel functions. A Bessel function of the $n$-th order is defined as

$$
J_{n}(x)=\frac{1}{2 \pi} \int_{0}^{2 \pi} \mathrm{e}^{-\mathrm{i} x \cos \varphi} \cos (\varphi n) \mathrm{d} \varphi .
$$

It will be remembered that the Bessel functions fulfill the following recurrence relations:

$$
\frac{\mathrm{d} J_{n}(x)}{\mathrm{d} x}=-\frac{n}{x} J_{n}(x)+J_{n-1}(x) .
$$


With (33), we calculate the derivative as

$$
\begin{gathered}
\left.\frac{\mathrm{d}\left[x J_{1}(a x)\right]}{\mathrm{d} x}=J_{1}(a x)+x\left[-\frac{1}{a x} J_{1}(a x) a+J_{0}(a x) a\right)\right] \\
=J_{1}(a x)-\frac{1}{a} J_{1}(a x) a+x J_{0}(a x) a=x J_{0}(a x) a,
\end{gathered}
$$

which is equivalent to the relation

$$
\left[x J_{1}(a x)\right]^{\prime}=J_{0}(a x) a x \text {. }
$$

On multiplying both terms of the preceding equation by $1 / a$, we arrive at

$$
\left[\frac{x}{a} J_{1}(a x)\right]^{\prime}=J_{0}(a x) x \text {. }
$$

On putting $r$ for $x$, and $G_{\|}$for $a$, we get

$$
\left[\frac{r}{G_{\|}} J_{1}\left(G_{\|} r\right)\right]^{\prime}=J_{0}\left(G_{\|} r\right) r
$$

Equation (32) (for $n=0$ ) enables us to rewrite the integral (31) in the following form:

$$
\widehat{\kappa}\left(\boldsymbol{G}_{\|}\right)=\frac{1}{\varepsilon_{b}} \delta\left(\boldsymbol{G}_{\|}\right)+\frac{2 \pi}{a^{2}}\left(\frac{1}{\varepsilon_{a}}-\frac{1}{\varepsilon_{b}}\right) \int_{0}^{R} r J_{0}\left(G_{\|} r\right) \mathrm{d} r .
$$

In dealing with the integral of (36) we consider two cases: 1 . for $\boldsymbol{G}_{\|}=0$

$$
\begin{aligned}
\widehat{\kappa}\left(\boldsymbol{G}_{\|}\right) & =\frac{1}{\varepsilon_{b}} \underbrace{\delta\left(\boldsymbol{G}_{\|}\right)}_{=1}+\frac{2 \pi}{a^{2}}\left(\frac{1}{\varepsilon_{a}}-\frac{1}{\varepsilon_{b}}\right) \int_{0}^{R} r \underbrace{J_{0}\left(G_{\|} r\right)}_{J_{0}(0)=1} \mathrm{~d} r \\
= & \frac{1}{\varepsilon_{b}}+\frac{2 \pi}{a^{2}}\left(\frac{1}{\varepsilon_{a}}-\frac{1}{\varepsilon_{b}}\right) \int_{0}^{r} r \mathrm{~d} r=\frac{1}{\varepsilon_{b}}+\left.\frac{2 \pi}{a^{2}}\left(\frac{1}{\varepsilon_{a}}-\frac{1}{\varepsilon_{b}}\right) \frac{r^{2}}{2}\right|_{0} ^{R} \\
= & \frac{1}{\varepsilon_{a}}+\frac{1}{\varepsilon_{b}}\left(1-\frac{\pi R^{2}}{a^{2}}\right)
\end{aligned}
$$

and 2. for $\boldsymbol{G}_{\|} \neq 0$ :

$$
\begin{aligned}
& =\frac{1}{\varepsilon_{b}} \underbrace{\delta\left(G_{\|}\right)}_{=0}+\left.\frac{2 \pi}{a^{2}}\left(\frac{1}{\varepsilon_{a}}-\frac{1}{\varepsilon_{b}}\right) \frac{r}{G_{\|}} J_{1}\left(G_{\|} r\right)\right|_{0} ^{R} \\
& =\left.\frac{2 \pi}{a^{2}}\left(\frac{1}{\varepsilon_{a}}-\frac{1}{\varepsilon_{b}}\right) \frac{r}{G_{\|}} J_{1}\left(G_{\|} r\right)\right|_{0} ^{R}=\frac{\pi R^{2}}{a^{2}}\left(\frac{1}{\varepsilon_{a}}-\frac{1}{\varepsilon_{b}}\right) \frac{2 J_{1}\left(G_{\|} R\right)}{\left(G_{\|} R\right)} .
\end{aligned}
$$

Having recourse to the notion of filling factor $f$ defined as the ratio of the area of the cross-section of the rod and the area of the cross-section of the elementary cell, i.e. for a square lattice with cylindrical rods

$$
f=\frac{\pi R^{2}}{a^{2}},
$$

we are justified in combining Eqs. (37) and (38) into the following single expression:

$$
\widehat{\kappa}\left(\boldsymbol{G}_{\|}\right)=\left\{\begin{array}{ccc}
\frac{1}{\varepsilon_{a}}+\frac{1}{\varepsilon_{b}}(1-f) & \text { for } & \boldsymbol{G}_{\|}=0, \\
\left(\frac{1}{\varepsilon_{a}}-\frac{1}{\varepsilon_{b}}\right) f \frac{2 J_{1}\left(G_{\|} R\right)}{\left(G_{\|} R\right)} & \text { for } \quad \boldsymbol{G}_{\|} \neq 0 .
\end{array}\right.
$$




\section{The case of double cylindrical rods}

In the present section we deal with a composite with the structure shown in Fig. 3. It differs from that studied in the preceding section in that the rods with a dielectric constant $\varepsilon_{a}$ (outer rods) are made to contain rods with a different

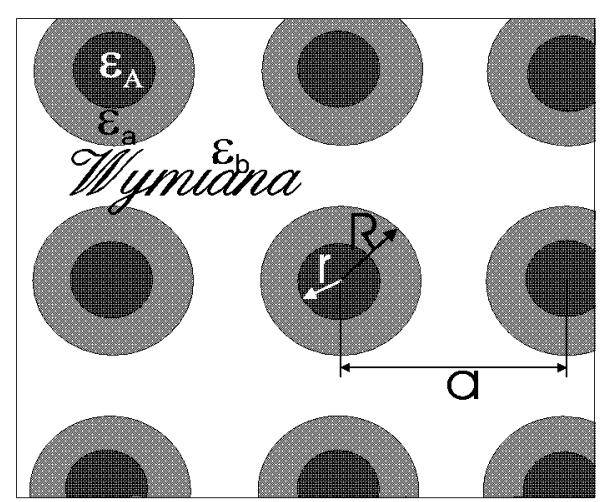

Fig. 3. The top view of the $2 \mathrm{D}$ square array of double circular rods; $\varepsilon_{A}$ and $\varepsilon_{a}$ denote dielectric constants of the inner and outer rods, respectively, $r$ and $R$ represent radii of respective rods.

constant $\varepsilon_{A}$ (inner rods). The dielectric permittivity function of this system as a whole takes the form

$$
\begin{aligned}
\frac{1}{\varepsilon\left(x_{\|}\right)} & =\frac{1}{\varepsilon_{b}}+\left\{\left[\frac{1}{\varepsilon_{a}}+\left(\frac{1}{\varepsilon_{A}}-\frac{1}{\varepsilon_{a}}\right) \sum_{l_{1}=-\infty}^{\infty} \sum_{l_{2}=-\infty}^{\infty} S_{r}\left[x_{\|}-x_{\|}(l)\right]-\frac{1}{\varepsilon_{b}}\right\}\right. \\
& \times \sum_{l_{1}=-\infty}^{\infty} \sum_{l_{2}=-\infty}^{\infty} S_{R}\left[x_{\|}-x_{\|}(l)\right],
\end{aligned}
$$

where

$$
\begin{aligned}
& S_{r}\left(x_{\|}\right)=\left\{\begin{array}{lll}
1 & \text { for } & \left|x_{\|}\right| \leq r \\
0 & \text { for } & \left|x_{\|}\right|>r
\end{array}\right. \\
& S_{R}\left(x_{\|}\right)=\left\{\begin{array}{lll}
1 & \text { for } & \left|x_{\|}\right| \leq R, \\
0 & \text { for } & \left|x_{\|}\right|>R
\end{array}\right.
\end{aligned}
$$

where $R$ and $r$ denote, respectively, the radii of the outer and inner rods.

We now insert the above defined permittivity function into Eq. (14):

$$
\begin{aligned}
\widehat{\kappa}\left(\boldsymbol{G}_{\|}\right) & =\frac{1}{a^{2}} \int_{0}^{a} \mathrm{~d} x_{1} \int_{0}^{a} \mathrm{~d} x_{2}\left(\frac{1}{\varepsilon_{b}}+\right. \\
+ & \left\{\left[\frac{1}{\varepsilon_{a}}+\left(\frac{1}{\varepsilon_{A}}-\frac{1}{\varepsilon_{a}}\right) \sum_{l_{1}=-\infty}^{\infty} \sum_{l_{2}=-\infty}^{\infty} S_{r}\left[x_{\|}-x_{\|}(l)\right]-\frac{1}{\varepsilon_{b}}\right\}\right.
\end{aligned}
$$




$$
\begin{aligned}
& \left.\times \sum_{l_{1}=-\infty}^{\infty} \sum_{l_{2}=-\infty}^{\infty} S_{R}\left[x_{\|}-x_{\|}(l)\right]\right) \mathrm{e}^{-\mathrm{i} \mathbb{G}_{\|} \cdot \boldsymbol{x}_{\|}} \\
& =\frac{1}{a^{2}} \int_{0}^{a} \mathrm{~d} x_{1} \int_{0}^{a} \mathrm{~d} x_{2} \frac{1}{\varepsilon_{b}} \mathrm{e}^{-\mathrm{i} \mathbb{U}_{\|} \|} \boldsymbol{x}_{\|} \\
& +\frac{1}{a^{2}} \int_{0}^{a} \mathrm{~d} x_{1} \int_{0}^{a} \mathrm{~d} x_{2}\left(\frac{1}{\varepsilon_{a}}-\frac{1}{\varepsilon_{b}}\right) S_{R}\left[x_{\|}-x_{\|}(l)\right] \mathrm{e}^{-\mathrm{i} \mathbb{G}_{\|} \| \cdot x_{\|}} \\
& +\frac{1}{a^{2}} \int_{0}^{a} \mathrm{~d} x_{1} \int_{0}^{a} \mathrm{~d} x_{2}\left(\frac{1}{\varepsilon_{A}}-\frac{1}{\varepsilon_{a}}\right) \sum_{l_{1}=-\infty}^{\infty} \sum_{l_{2}=-\infty}^{\infty} S_{r}\left[x_{\|}-x_{\|}(l)\right] \\
& \times S_{R}\left[x_{\|}-x_{\|}(l)\right] \mathrm{e}^{-i \mathbb{G}_{\|} \cdot x_{\|}} .
\end{aligned}
$$

Above, the first integral has a structure analogous to that of the integral (19); accordingly, we make use of the results obtained in the respective section, leading to

$$
\frac{1}{a^{2}} \frac{1}{\varepsilon_{b}} \int_{0}^{a} \mathrm{~d} x_{1} \int_{0}^{a} \mathrm{~d} x_{2} \mathrm{e}^{-\mathrm{i}\left(\mathbb{U}_{\|} \|_{\|}\right)}=\frac{1}{\varepsilon_{b}} \delta\left(\boldsymbol{G}_{\|}\right) .
$$

The remaining two integrals are of the same type as the integral (20). We note that the product of the functions $S_{r}\left(\boldsymbol{x}_{\|}\right)$and $S_{R}\left(x_{\|}\right)$(as defined in (42) and (43)) takes the value

$$
S_{r}\left[\boldsymbol{x}_{\|}-x_{\|}(l)\right] S_{R}\left[x_{\|}-x_{\|}(l)\right] \equiv S_{r}\left[x_{\|}-x_{\|}(l)\right] ;
$$

thus, we can write the expression for the coefficient $\widehat{\kappa}\left(\boldsymbol{G}_{\|}\right)$in the case under consideration in the form

$$
\widehat{\kappa}\left(\boldsymbol{G}_{\|}\right)=\frac{1}{\varepsilon_{c}} \delta_{\mathbb{G}^{t} \|, 0}+\left(\frac{1}{\varepsilon_{a}}-\frac{1}{\varepsilon_{b}}\right) f \frac{2 J_{1}\left(G_{\|} R\right)}{\left(G_{\|} R\right)}+\left(\frac{1}{\varepsilon_{A}}-\frac{1}{\varepsilon_{a}}\right) F \frac{2 J_{1}\left(G_{\|} r\right)}{\left(G_{\|} r\right)},
$$

where, respectively, $F$ and $f$ are the filling factors of the inner and outer cylinders defined as follows:

$$
\begin{aligned}
& F=\frac{\pi r^{2}}{a^{2}}, \\
& f=\frac{\pi R^{2}}{a^{2}} .
\end{aligned}
$$

Finally, we write (46) in a form similar to (40):

$$
\widehat{\kappa}\left(\boldsymbol{G}_{\|}\right)= \begin{cases}\left(\frac{1}{\varepsilon_{a}}-\frac{1}{\varepsilon_{b}}\right) f \frac{2 J_{1}\left(G_{\|} R\right)}{\left(G_{\|} R\right)}+\left(\frac{1}{\varepsilon_{A}}-\frac{1}{\varepsilon_{a}}\right) F \frac{2 J_{1}\left(G_{\|} r\right)}{\left(G_{\|^{r}}\right)} & \text { for } \quad \boldsymbol{G}_{\|} \neq 0, \\ \frac{1-f}{\varepsilon_{b}}+\frac{1}{\varepsilon_{a}} f+\left(\frac{1}{\varepsilon_{A}}-\frac{1}{\varepsilon_{a}}\right) F & \text { for } \quad \boldsymbol{G}_{\|}=0 .\end{cases}
$$




\section{Calculated photonic energy spectra}

The essential numerical problem is that of the convergence of our results. It arises due to the fact that the equations whence we determine the photonic spectra are well determined for a crystal of infinite size. Thus, we deal with a crystal with an infinitely great number of sites on the original lattice and consequently an infinite reciprocal lattice over the sites of which summation runs. In our numerical work we have recourse to a simplification restricting summation to a finite number of sites of the reciprocal lattice. In numerical practice we cannot avoid compromising between the degree of convergence obtained and the achievable time of the computer work. On the basis of our experience and that of others [27] we chose $N=441$ as ensuring one-percent convergence.

We now proceed to a detailed analysis of the photonic energy band spectra obtained for two-dimensional composite crystal constructed with cylindrical rods (simple and double). We shall analyze the mutual relations between the shape, the inner structure, and the size of the rods and their dielectric surroundings as well as the width and disposition of the energy gaps. We start from a discussion of structures composed of single dielectric cylindrical rods. Figures 4 and 5 show the mechanism of formation of photonic energy gaps versus the filling factor $f$ for $n=0$ (at $n=0$ the spectra are independent of the ratio $d / a$; that is to say, they are insensitive to the separation of surfaces [27]). For small $f \leq 0.02$, we observe no gaps, an increase in $f$ up to 0.05 leads to the formation of the first two gaps (and a lowering of the individual energy branches). A further increase in $f$ opens the third gap and causes a further lowering of gaps. Concerning the width of the energy gaps, we shall consider only the case when the filling factor $f$ fulfills the relation $0<f<0.79$, i.e. when the dielectric rods are well "separated". Figures $5 \mathrm{a}$ and $\mathrm{b}$ show the widths of the first, second, and third energy gaps versus the filling factor $f$ and permittivity $\varepsilon_{a}$. A gradual increasing $f$ is found to open a gap only if a certain critical value is exceeded (different for each of the gap). The gap width then increases steeply and reaches its maximum, afterwards we observe a slow narrowing of the gap down to its disappearance. For each of the gap there exists a characteristic value of $f$ followed by a steep increase in width. These characteristic values of $f$ are attained successively by the successive gaps.

We now proceed to the study of energy spectra of structures composed of double dielectric rods. Let us now elucidate the effect of the inner cylinder on the width of the gap. Figure 6 visualizes the difference between the width of the first gap as obtained for the structure of double cylinders and the width of the same gap obtained in the model with simple cylinders (for the same values of the filling factor and the two permittivities), the variable parameters being the value of the filling factor of the inner rod and its dielectric permittivity. We note that for the gap, the gain from the enrichment of the structure by the inner rod is of the order of 100 percent when adding an inner cylinder with a permittivity higher than that of the outer cylinder (cf. Figs. $7 \mathrm{~b}$ and c), but also we even observe a small increase in the gap width when an inner cylinder has permittivity lower than the outer one (cf. Figs. $7 \mathrm{a}$ and $\mathrm{b}$ ). 


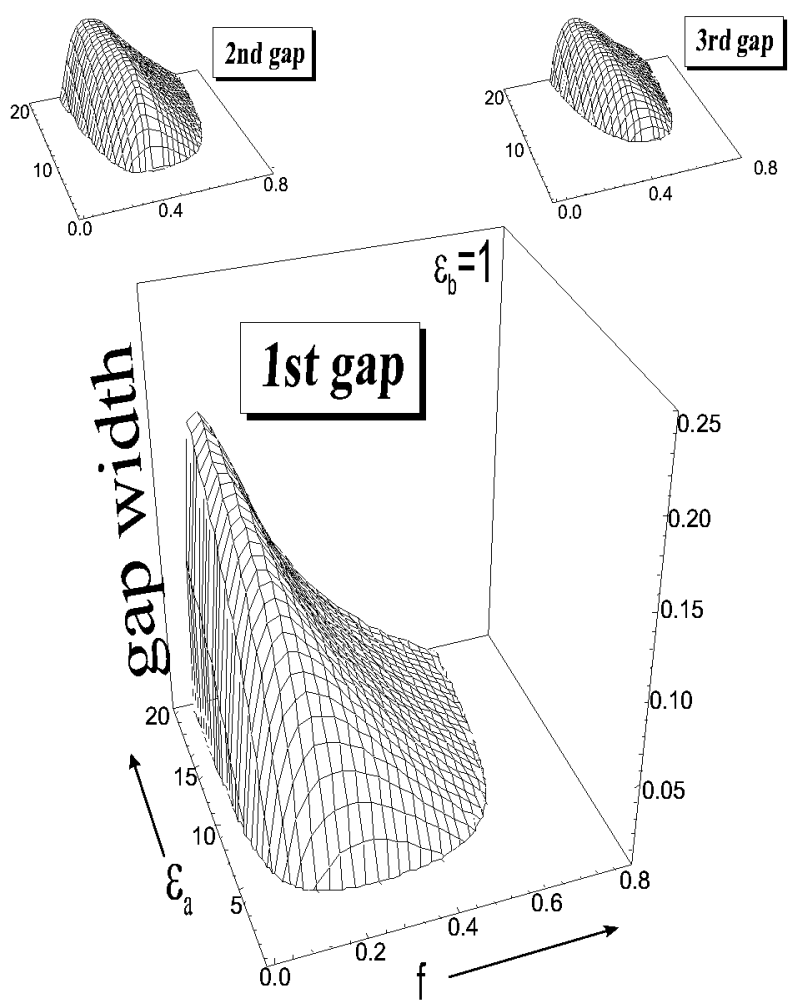

Fig. 4. The width (in units of the reduced frequency) of the 1st, 2nd, and 3rd gap vs. the filling fraction, $f$, and the dielectric constant $\varepsilon_{a}$ (for a $2 D$ photonic crystal composed of single dielectric rods).
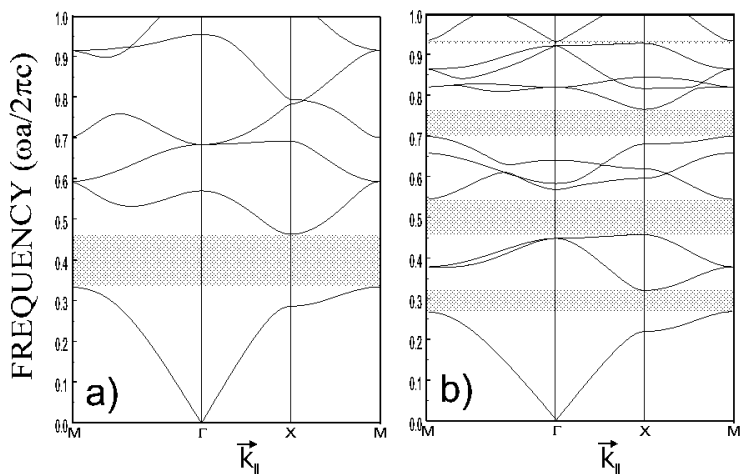

Fig. 5. Frequency gaps calculated for photonic crystal composed of single rods (for $N=441$, the number of shortest vectors of the reciprocal lattice). The respective structure parameters are: (a) $f=0.16, \varepsilon_{a}=10$ and $\varepsilon_{b}=1$; (b) $f=0.38, \varepsilon_{a}=10$ and $\varepsilon_{b}=1$. The two-dimensional wave vector $\boldsymbol{k}_{\|}$(in units $2 \pi / a$ ) extends over the first Brillouin zone. 


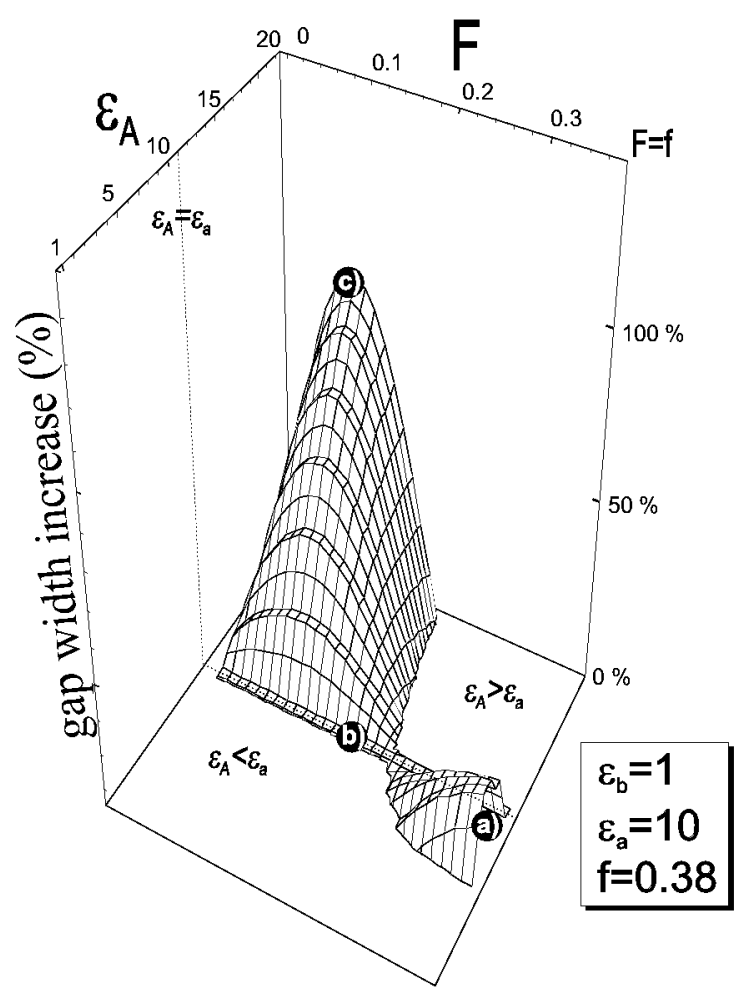

Fig. 6. The increase in the width of the first (lowest) photonic frequency gap caused by an introduction of an additional inner cylinder (its parameters $\varepsilon_{A}$ and $F$ are treated as variables). It is interesting to note that the gap grows wider even in the region where $\varepsilon_{A}$ is smaller than $\varepsilon_{a}$.
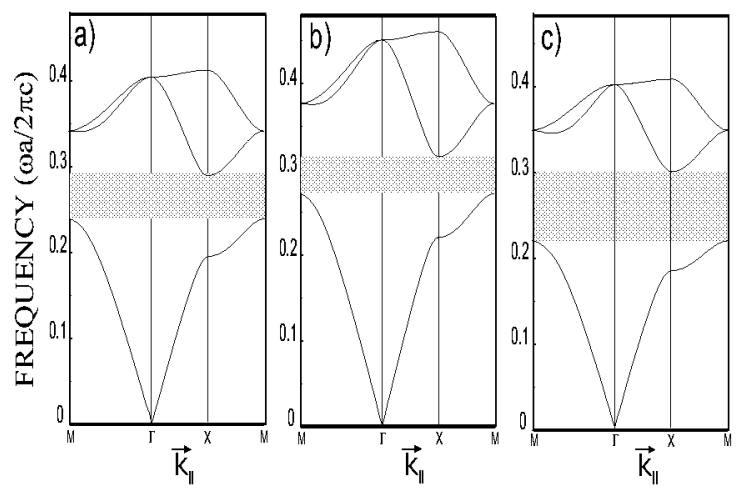

Fig. 7. The lowest frequency gap in the photonic crystal composed of double rods; parts (a), (b), and (c) correspond to particular structure parameters indicated in Fig. 6. Note that case (b) corresponds in fact to the intermediate case of a single rod structure. 


\section{Conclusions}

We studied the properties of a two-dimensional (surface-bounded) photonic macrocrystal composed of cylindrical dielectric rods (the limiting surfaces were perpendicular to the axes of the cylinders). We have determined the parameters of the structure best favoring the emergence of absolute photonic energy gaps. In particular, we dealt with the changes in energy spectrum caused by the insertion of a foreign rod with different dielectric constant into the original rods, thus giving rise to a system of double rods. We found that the insertion of a foreign (inner) cylindrical rod increases the width of the absolute energy gaps. In the next paper we intend to perform a similar study for the case of rods (inner and/or outer) having rectangular cross-sections.

\section{Acknowledgments}

The present work was supported by grant No. 2 PO3B 07916 from the Committee for Scientific Research.

\section{References}

[1] E. Yablonovitch, Phys. Rev. Lett. 58, 2059 (1987).

[2] E. Ozbay, A. Abeyta, G. Tuttle, M. Tringides, R. Biswas, C.T. Chan, C.M. Soukoulis, K.M. Ho, Phys. Rev. B 50, 1945 (1994).

[3] E. Ozbay, E. Michel, G. Tuttle, R. Biswas, K.M. Ho, J. Bostak, D.M. Bloom, Opt. Lett. 19, 1155 (1994).

[4] J.S. McCalmont, M.M. Sigalas, G. Tuttle, K.M. Ho, C.M. Soukoulis, Appl. Phys. Lett. 68, 2759 (1996).

[5] J.D. Joannopoulos, R.B. Meade, J.N. Winn, Photonic Crystals: Molding the Flow of Light, Princeton University Press, Princeton 1996.

[6] H.S. Sozuer, J.W. Haus, J. Opt. Soc. Am. B 10, 296 (1993).

[7] J.B. Pendry, J. Phys., Condens. Matter 8, 1085 (1996).

[8] A. Rosenberg, R.J. Tonucci, H.B. Lin, E.L. Shirley, Phys. Rev. B 54, 5195 (1996).

[9] M.J. Ries, E.I. Chen, N. Holonyak, Appl. Phys. Lett. 68, 2035 (1996).

[10] T. Baba, M. Koma, Jpn. J. Appl. Phys. 34, 1405 (1995).

[11] Photonic Band Gap Materials, Ed. C.M. Soukoulis, Kluwer Academic, Dordrecht 1996.

[12] J.N. Winn, R.D. Meade, J.D. Joannopoulos, J. Mod. Opt. 41, 257 (1994).

[13] R. Padjen, J.M. Gerard, J.Y. Marzin, J. Mod. Opt. 41, 295 (1994).

[14] T. Baba, T. Matsuzaki, Jpn. J. Appl. Phys. 34, 4496 (1995).

[15] D. Cassagne, C. Jouanin, D. Bertho, Phys. Rev. B 53, 7134 (1996).

[16] K. Sakoda, Phys. Rev. B 52, 8992 (1995).

[17] M. Plihal, A. Shambork, A.A. Maradudin, P. Sheng, Opt. Commun. 80, 199 (1991).

[18] M. Plihal, A.A. Maradudin, Phys. Rev. B 44, 8565 (1991). 
[19] P.R. Villeneuve, M. Piché, J. Opt. Soc. Am. A 8, 1296 (1991).

[20] R. Hillebrand, W. Hergert, W. Harms, Phys. Status Solidi B 217, 981 (2000).

[21] S.L. McCall, P.M. Platzman, R. Dalichaouch, D. Smith, S. Schultz, Phys. Rev. Lett. 66, 2017 (1991).

[22] R.D. Meade, K.D. Brommer, A.M. Rappe, J.D. Joannopoulos, Appl. Phys. Lett. 61, 495 (1992).

[23] X.H. Wang, B.Y. Gu, Z.Y. Li, G.Z. Yang, Phys. Rev. B 60, 11417 (1999).

[24] M. Qiu, S. He, J. Opt. Soc. Am. B 17, 1027 (2000).

[25] M. Qiu, S. He, Phys. Rev. B 60, 10610 (1999).

[26] X. Zhang, Z.Q. Zhang, Phys. Rev. B 61, 9847 (2000).

[27] A.A. Maradudin, A.R. McGurn, J. Opt. Soc. Am. B 8, 307 (1993). 